\title{
Mantenedor de espacio funcional elaborado con corona de NuSmile.
}

\section{Elaborated functional space maintainer with zirconia NuSmile crown.}

\author{
Kevin Juárez Casanova*
}

\section{RESUMEN}

La caries de la infancia temprana, al igual que otras formas de caries, se caracteriza por ser multifactorial, infecciosa y de rápida evolución. Puede presentarse como lesiones cavitadas o no cavitadas, se diagnostica principalmente en menores de seis años en la superficies de los dientes anterosuperiores, debido a que son los primeros órganos dentales en erupcionar y tienen mayor contacto con el estímulo cariogénico. En casos severos pueden llegar a ocasionar la pérdida de órganos dentales, influyendo en la salud general del paciente y repercutiendo en su autoestima y desarrollo psicosocial. Para devolver las características y funciones perdidas en ausencia de algún órgano dental, se recurre a sustituirlo mediante aparatología, mayormente en forma de un mantenedor de espacio funcional. Las necesidades estéticas individuales de cada caso generan diversas alternativas para adaptarnos a la situación del paciente.

Palabras clave: Caries de la infancia temprana, mantenedor de espacio, corona de zirconia.

\section{ABSTRACT}

Early childhood caries, like other forms of caries, is characterized by being multifactorial, infectious, and rapidly evolving. It can present as cavitated or uncavitated lesions, it is mainly diagnosed in children under six years of age on the surfaces of the anterior superior teeth, because they are the first dental organs to erupt and have greater contact with the cariogenic stimulus. In severe cases they can cause the loss of dental organs, influencing the general health of the patient and impacting on their self-esteem and psychosocial development. In order to restore the lost features and functions in the absence of any dental organ, it is resorted to by means of appliances, mostly in the form of a functional space maintainer. The individual aesthetic needs of each case generate various alternatives to adapt to the patient's situation.

Keywords: Early childhood caries, space maintainer, zirconia crown.

\section{INTRODUCCIÓN}

L a Asociación Internacional de Odontología Pediátrica (IAPD), mediante la declaración de Bangkok, define la caries de la infancia temprana (CIT) como la presencia de una o más lesiones (cavitadas o no cavitadas), perdidas o restauradas (debido a caries) en cualquier órgano dental primario que se halle en niños menores a seis años. ${ }^{1}$

Al igual que otras formas de caries, se considera azúcar y biofilm-dependiente, infecciosa, de rápida evolución y multifactorial, que da como resultado el desequilibrio del proceso de remineralización y desmineralización de los tejidos duros del diente. ${ }^{1,2}$

Se inicia al desmineralizarse la superficie del esmalte de forma drástica por la producción de ácidos producidos por la alteración de $\mathrm{pH}$ menor a 5.0, a causa de la presencia de hidratos de carbono metabolizados por el biofilm, unida a la exposición frecuente y prolongada de alimentos con alto contenido de azúcar, situación que está directamente relacionada con la alimentación del niño

\footnotetext{
* Residente, Especialidad de Odontología Pediátrica. Universidad Anáhuac Mayab. Mérida, Yucatán, México.

Recibido: 27 de julio de 2020. Aceptado: 18 de julio de 2021.

Citar como: Juárez CK. Mantenedor de espacio funcional elaborado con corona de NuSmile. Rev ADM. 2021; 78 (4): 229-234. https://dx.doi. org/10.35366/101078
} 
a partir de su nacimiento, además de malos hábitos de higiene bucal, la colonización de Streptococcus mutans, flujo salival, patrón de erupción dental y alteraciones como defectos de estructura que hagan más susceptibles al órgano dental. Otros factores de riesgo asociados con la CIT son: económicos, culturales y conductuales. ${ }^{2-5}$

La edad y frecuencia con la que se le presentan alimentos ricos en azucares libres al niño como frutas, jarabes, jugos y concentrados influyen en el desarrollo de caries dental, además, el consumo prolongado y nocturno del biberón más allá de los 12 meses también se asocia a la CIT. Debido a ello, en el pasado se le conocía como «caries de biberón» o «síndrome de biberón». ${ }^{5}$

Es de suma importancia recalcar que la leche materna no es cariogénica por sí misma debido a la acción de la lactoferrina (quelato de hierro, bactericida que impide la nutrición de Streptococcus mutans) a menos que haya algún otro azúcar fermentable en la dieta. ${ }^{6}$

Los incisivos maxilares son los más propensos a adquirir $\mathrm{CIT}$, ya que son los primeros órganos dentales en erupcionar y los que tienen el primer contacto con los estímulos cariogénicos. ${ }^{3}$ Así como los traumatismos dentales, ausencias de origen congénito, erupción ectópica o malformaciones dentales pueden ser factores de pérdida prematura de órganos dentarios en pacientes preescolares, la caries de la infancia temprana también puede traer como consecuencia en casos severos la pérdida de un órgano dental. ${ }^{4}$

La caries de la infancia temprana también influye en la salud general del paciente, como retraso en el crecimiento y desarrollo, pudiendo presentar un peso y estatura inadecuadas, ${ }^{2}$ los efectos que se producen al perder incisivos maxilares traen consigo alteraciones de desarrollo orofacial, de fonación, estética, nutricionales, además de favorecer la aparición de hábitos orales de succión labial y digital o de interposición lingual que fomentará el desarrollo de una mordida abierta. ${ }^{7-9}$

La baja aceptación estética al presentar alguna ausencia repercute en la psicología y autoestima del paciente, genera timidez e incluso depresión, el niño no sonríe y se vuelve introvertido, disminuyen sus relaciones y comunicación, influye en su vida y actividades cotidianas, por lo que un tratamiento que le devuelva la función y estética puede mejorar la calidad de vida. 9,10

El tratamiento de los órganos dentales severamente deteriorados plantea un desafío para el especialista en odontología infantil, ya que se toman en cuenta cuatro aspectos importantes: el manejo de conducta, preservación de estructura, la satisfacción de los padres y aunque por lo general se hace a un lado, también es necesario tomar en cuenta la opinión del paciente, ya que es él quien va a ser el receptor de la restauración. La percepción de un paciente pediátrico varía dependiendo de la edad, género, educación y factores socioculturales. ${ }^{11}$

A diferencia del sector posterior, en la ausencia prematura de los incisivos superiores no es frecuente ni común la pérdida de espacio por mesialización de los órganos dentarios adyacentes; sin embargo, sí podría haber extrusión de antagonistas. Por lo anterior, es importante colocar algún dispositivo como mantenedor de espacio para devolver función, permitir un correcto desarrollo del arco dentario y otorgar estética y armonía, con esto sustituimos los órganos afectados por la caries de la infancia temprana. El mantenedor de espacio funcional es ideal y de fácil adaptación para el paciente en largos periodos de uso, los más mencionados en la literatura son aquéllos que cuentan con bandas ortodóncicas y dientes de acrílico para imitar los órganos ausentes y tener resultados favorables en cuestiones de estética y satisfacción para los padres y el paciente. No requieren preparación invasiva y no lesiona tejidos periodontales. $4,7,12$

Existe un periodo de estabilidad en el crecimiento y desarrollo en sentido sagital y transversal de los maxilares entre los tres y cinco años, aunque es importante considerar el seguimiento que se debe dar con estos mantenedores, ya que hay posibilidad de que otros factores influyan alterando este periodo. Un correcto control incluye el reemplazo constante del aparato protésico cuando es necesario. ${ }^{7}$

Las coronas NuSmile se introdujeron en 1991, en un inicio eran coronas de acero inoxidable con una cubierta de color similar al de un diente, hoy en día son coronas fabricadas de zirconia (cristal de dióxido de zirconio) y ofrecen beneficios como retención, adhesión celular y resistencia a desgaste de antagonistas y filtración marginal, además de una impecable estética, sin contar que también otorgan seguridad y autoestima, modificando por completo la sonrisa, aunque tienen sus indicaciones precisas y algunas desventajas son principalmente el costo, su ajuste es complicado ya que es el diente el que se adapta a la corona, no la corona al diente, como sucede con las coronas de acero cromo. Se encuentran en el mercado para toda la dentición infantil y para primeros molares permanentes. ${ }^{3,13-16}$

El objetivo de este escrito es evaluar una alternativa al mantenedor de espacio funcional mediante el uso de corona de zirconia NuSmile, en lugar de un diente de acrílico convencional debido a las necesidades estéticas del paciente y sus tutores. 


\section{PRESENTACIÓN DEL CASO}

Paciente femenino de cuatro años de edad originaria de Cancún, Quintana Roo, acude a la clínica del postgrado de Odontología Pediátrica de la Universidad Anáhuac Mayab en octubre de 2019, acompañada de sus tíos, quienes no refieren antecedentes heredofamiliares ni personales patológicos, sin alteraciones en cabeza y cuello, cuyo motivo de consulta fue: «quitarle corona blanca, porque se ve fea». También refieren que la paciente se ha vuelto retraída, tímida y casi no sonríe por cuestión estética; sin embargo, se les comentó que el verdadero problema se hallaba en evidente lesión cariosa del órgano dental 61.

\section{Diagnóstico y plan de tratamiento}

Al realizar exploración clínica, se observa dentición primaria completa de acuerdo con la edad. Tejidos blandos se encuentran sin alteraciones. En el sector anterosuperior se diagnosticó caries de la infancia temprana al observar clínicamente lesiones blancas no cavitadas, presencia
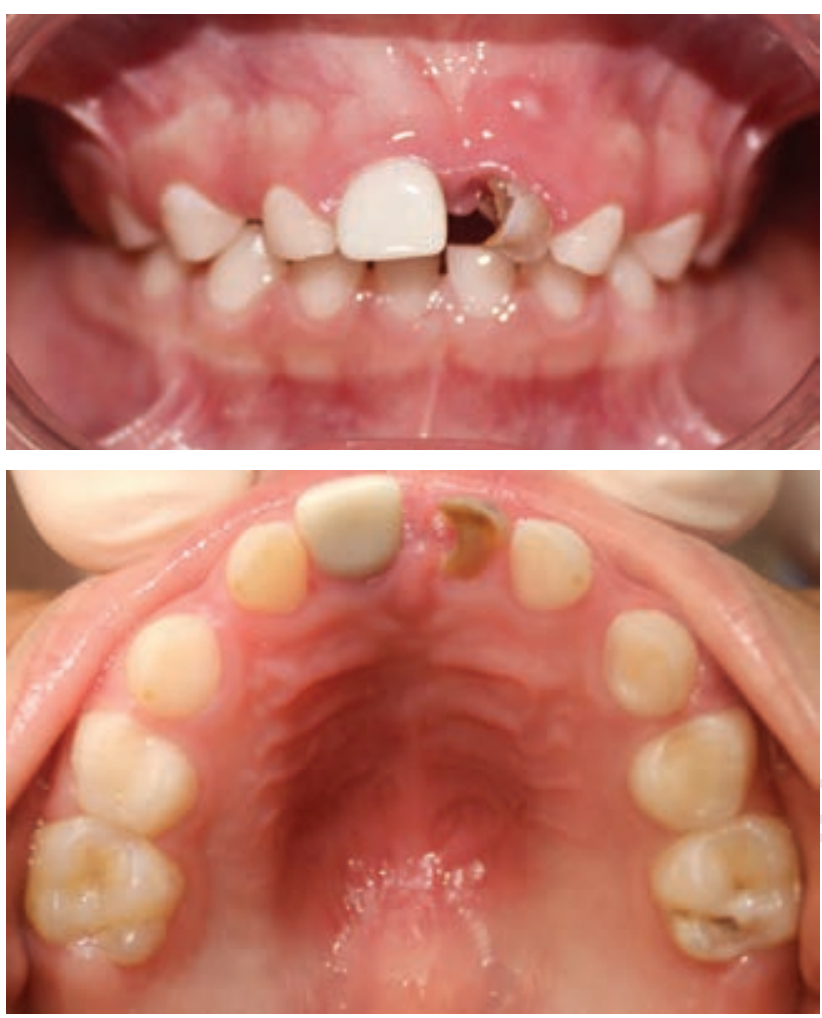

Figura 1: Fotografías iniciales del paciente.

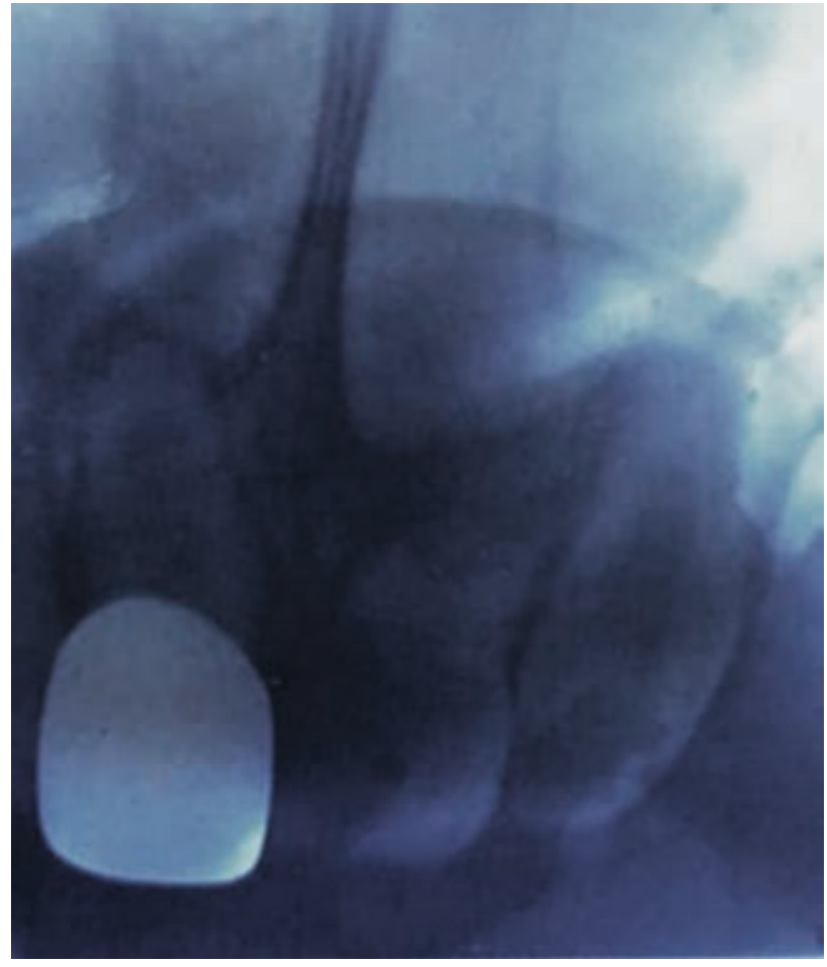

Figura 2: Radiografía periapical.

de restauración con corona de zirconia NuSmile en el órgano dental (OD) 51 y en el OD 61 se observa lesión cariosa severa asintomática, con aparente antecedente de trauma debido a sus características físicas como discromía, movilidad y pérdida de estructura coronal, así como remanente de absceso localizado en la zona superior de la gingiva (Figura 1).

Al hacer una valoración radiográfica, se observa ausenscia de tratamiento de conductos en el OD 51, así como reabsorción interna radicular en el OD 61, zona radiolúcida comprometiendo cámara pulpar y evidente pérdida de estructura coronal (Figura 2), por lo que se decide extraer el órgano dental como única alternativa de tratamiento.

Posterior a la exodoncia del OD 61, se planea colocar mantenedor de espacio para devolver función y estética a la paciente, al observar que habría un contraste importante de color entre el diente de acrílico del mantenedor, la restauración del OD 51 con corona de zirconia NuSmile y el OD 62 con su estructura natural, se decide confeccionar la prótesis a partir de una corona de zirconia NuSmile mimetizándose con la restauración adyacente para mejorar el aspecto. 


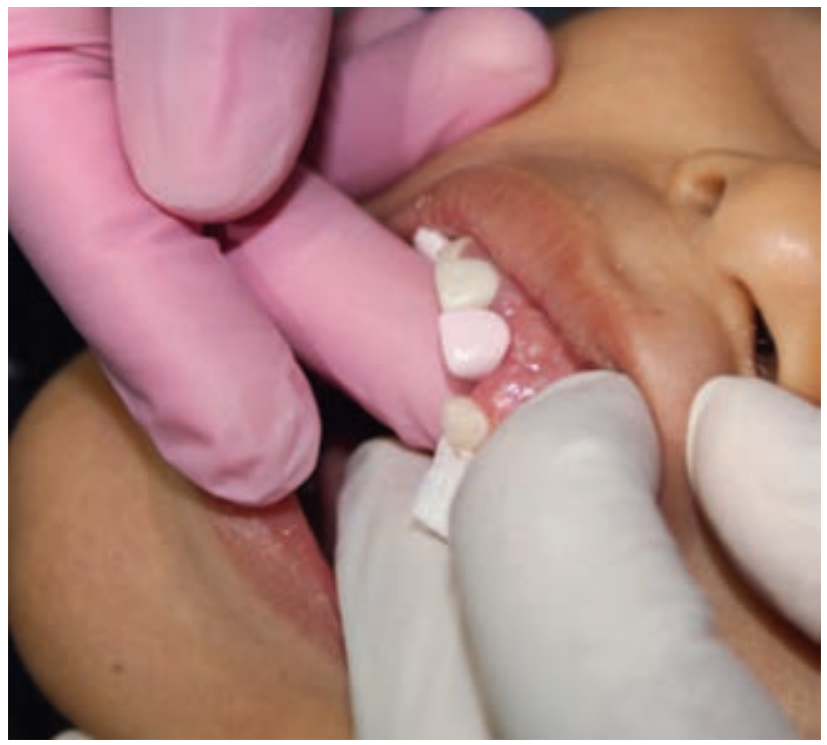

Figura 3: Selección y prueba con corona Try In de zirconia.

\section{Tratamiento}

Como parte de su rehabilitación, se confeccionará mantenedor de espacio funcional para sustituir la ausencia del incisivo central superior izquierdo y aplicación de barniz de flúor para control de las lesiones blancas no cavitadas.

Se aplica base de acrílico rosa en la zona desdentada y se selecciona la corona de zirconia NuSmile del kit para piezas anteriores, primero se probó directamente en el paciente con la corona Try In rosada, evitando contaminación cruzada y se evalúa el espacio mesiodistal y altura (Figura 3), después se comparó color y tamaño con el OD 51, de esta forma se seleccionó la corona A2L y se trasladó al modelo de estudio. Sin embargo, al probarla sobre la retención y base de acrílico, se observó que no estaban a la misma altura, por lo que se decide hacer remoción selectiva con fresa de desgaste para zirconia en la zona cervical de la corona hasta adaptarla totalmente.

Una vez realizadas las pruebas, se procede a embeber el interior de la corona con acrílico transparente, posicionándola en la retención del mantenedor (Figura 4), se pule cuidadosamente el arco, bandas y acrílico. Para finalizar, se realiza cementación de la prótesis con ionómero de vidrio (Ketac ${ }^{\mathrm{TM}}$ Cem Easy Mix ${ }^{\mathrm{TM}}$ ) (Figura 5).

Se hizo énfasis a los tutores del caso sobre la importancia de llevar una correcta higiene bucal, cuidados del mantenedor de espacio, modificación de dieta, así como prevención y control de las lesiones blancas que se encuentran en la zona anterior.
La paciente tuvo un cambio significativo con respecto a su estado de ánimo y personalidad, ya que se le había devuelto la armonía a su sonrisa, mejorando con esto su autoestima.

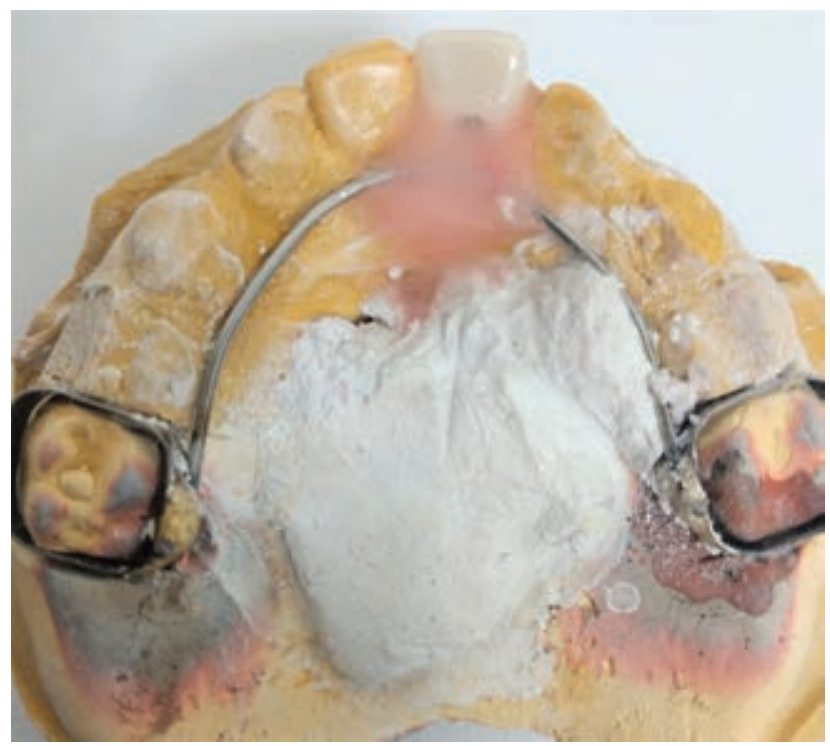

Figura 4: Elaboración del mantenedor de espacio.
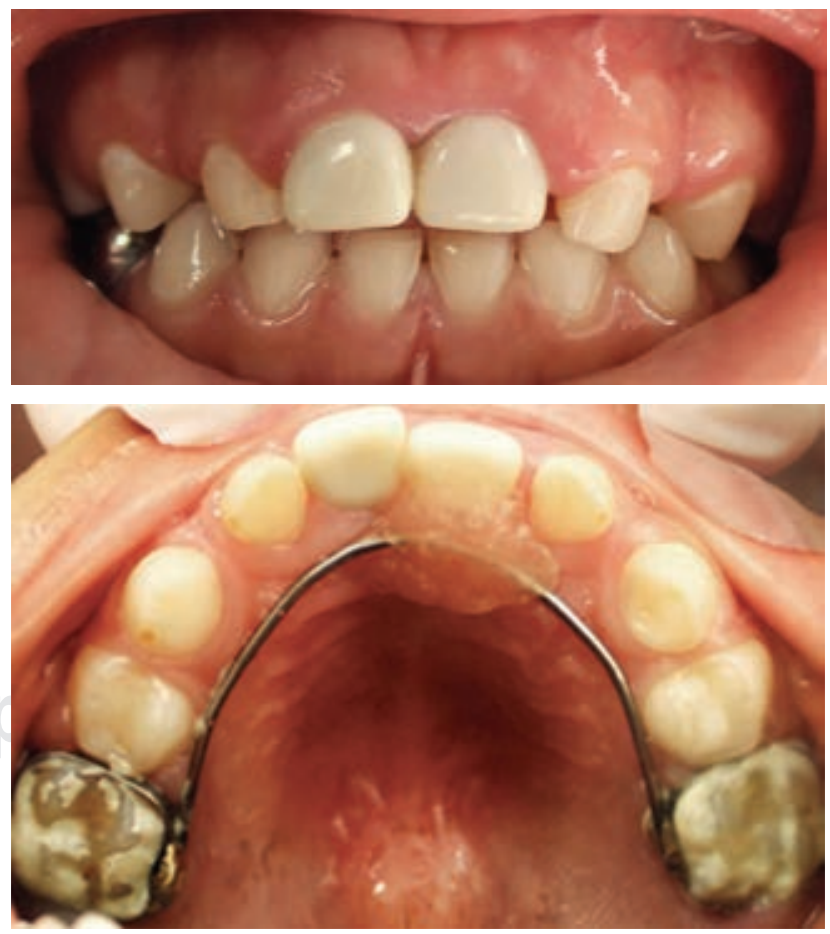

Figura 5: Fotografías finales de la paciente. 


\section{DISCUSIÓN}

Como se mencionó, la presencia integral de la dentición decidua es importante para un correcto estado de salud, no sólo bucodental, pues también puede repercutir en la salud general, estado nutricional y desarrollo psicosocial de un niño en edad preescolar, así como consecuencias en el habla, fonación y autoestima. Una de las causas más frecuentes cuando se trata de la pérdida prematura del órgano dental es la caries de la infancia temprana, como en el caso presentado. ${ }^{4}$

Esta patología se caracteriza por presentarse en menores de 71 meses de edad y principalmente se ve reflejada en los órganos dentales de la zona anterior. Las consecuencias en casos severos de caries de la infancia temprana son las extracciones de los órganos dentarios afectados. Al ser extraídos los dientes, propicia el desarrollo de hábitos orales, problemas estéticos y un déficit masticatorio. $^{3}$

El uso de mantenedores de espacio para reemplazar órganos dentarios prematuramente perdidos es una cuestión interesante, se indican cuando hay ausencia de uno o más. El odontólogo debe diseñar y construir la prótesis cumpliendo una serie de criterios que devuelvan la correcta función y estética, aplicando conocimientos sobre el correcto crecimiento y desarrollo de las arcadas, así como la cronología de la dentición, cumpliendo esto se valora qué tan factible es el uso de una prótesis. ${ }^{17}$

En la actualidad, la demanda en tratamientos estéticos en consulta dental ha ido en aumento y la odontopediatría no se queda lejos de estas necesidades, debido a esto se han desarrollado materiales como las coronas de zirconia, pues tienen excelentes cualidades estéticas y funcionales. ${ }^{16}$

Las coronas de zirconia de la casa NuSmile se han convertido en uno de los materiales restauradores mejor evaluados por odontopediatras para rehabilitar órganos dentales, las principales características para las cuales se elige su aplicación es por la capacidad de soportar fuerzas masticatorias sin fracturarse, así como la alta estética que ofrece. ${ }^{13}$

Por lo general, el mantenedor de espacio funcional se elabora con dientes de acrílico; sin embargo, de acuerdo con lo descrito por Gutiérrez y colaboradores, es totalmente factible el uso de coronas NuSmile como alternativa para la elaboración de mantenedor de espacio funcional en el sector anterior como explica en un caso clínico. ${ }^{18}$ De esta forma resulta una prótesis más armónica para la paciente que ya venía con una restauración de corona de zirconia NuSmile en el OD adyacente.
Una de las desventajas del uso de coronas de zirconia para la restauración de OD lesionados en pacientes infantiles es el alto costo que pudiese significar, ${ }^{16}$ en el caso de la paciente se recurrió a ellas para poder equilibrar el contraste que pudiese generar tres tonalidades diferentes: el color del diente natural, el diente de acrílico y el de la corona de zirconia ya existente, y así tener mejores resultados con respecto a la satisfacción del paciente y tutores.

Es importante no dejar de lado las citas periódicas de control para evaluar cambios que pudiesen modificar algún aspecto de la prótesis, además de dar instrucciones de higiene y cuidado oral y seguimiento durante el futuro recambio eruptivo. ${ }^{19}$

Tal como se menciona en la literatura, devolver la estética al finalizar el tratamiento por medio del mantenedor de espacio funcional para sustituir el órgano dental lesionado, propició un cambio en la conducta del paciente, pasó de ser tímida, introvertida y evitando entablar comunicación verbal a alguien con más confianza, actitud positiva y que buscaba cualquier momento para mostrar su sonrisa, demostrando una total satisfacción. ${ }^{9}$

\section{CONCLUSIONES}

Los resultados obtenidos al finalizar la secuencia de tratamiento y colocando el mantenedor de espacio funcional, sustituyendo diente de acrílico por una corona de zirconia NuSmile, para devolver función y estética debido a pérdida prematura de un órgano dental anterior con antecedentes de caries de la infancia temprana en la arcada superior han sido favorables para el paciente, tutores y el odontopediatra mismo, aunado a esto, se obtienen mejoras en la conducta del paciente, desenvolvimiento psicológico y social en su entorno.

\section{AGRADECIMIENTOS}

Al postgrado de Odontología Pediátrica de la Universidad Anáhuac Mayab.

Dr. Armando González Solís, Coordinador y Dr. Héctor Martínez Menchaca, Profesor del mismo postgrado.

\section{Dr. Eduardo Cantú Solís.}

\section{REFERENCIAS}

1. Pitts N, Baez R, Diaz-Guallory C et al. Early childhood caries: IAPD Bangkok declaration. Int J Paediatr Dent. 2019; 9: 384-386.

2. Guerrero-Castellón MP, Galeana-Ramírez MG, Corona-Zavala AA. Caries de la infancia temprana: medidas preventivas y rehabilitación. Rev Odontol Latinoam. 2012; 4 (1): 25-28. 
3. Medrano-González IC, Esparza-Ramos F, Rangel-Padilla EE, YáñezAcosta MF, Ramírez-Peña HA. Restauración estética del sector anterior en un paciente pediátrico. Rev Tamé. 2018; 7 (19): 733-737.

4. González-Vázquez V, Olivares-Campos O, Pedroza-Uribe I, AlcaláSánchez J, Velázquez-Jiménez Y. Rehabilitación con mantenedor de espacio funcional en el sector anterior en un paciente pediátrico. Rev Tamé. 2017; 6 (17): 625-629.

5. Tinanoff N, Baez RJ, Diaz Guillory C et al. Early childhood caries epidemiology, aetiology, risk assessment, societal burden, management, education, and policy: global perspective. Int Paediatr Dent. 2019; 29: 238-248.

6. Cantú-Solís E, Loya LME, Sanín LH et al. Caracterización de la flora bucal según patrón de amamantamiento en un grupo de madres e hijos de la Ciudad de Chihuahua, Chihuahua. Lactancia y flora patógena oral. Rev ADM. 2015; 72 (3): 146-152.

7. Zaror C, Hope López B, Díaz Melendez J, Jans Muñoz A. Prótesis fija con sistema tubo-barra en odontopediatría: reporte de un caso clínico de 12 meses de seguimiento. Rev Clin Periodoncia Implantol Rehabil Oral. 2015; 8 (3): 239-243.

8. Moreno AS, Pedraza CG, Lara CE. Mantenedor de espacio en pérdida prematura de órganos dentarios en dentición mixta. Reporte de un caso clínico. Rev ADM. 2011; 68 (1): 30-34.

9. Casafont AA, Chan L, Brenes A. Rehabilitación protésica en pacientes pediátricos. Caso reporte. Odovtos-Int J Dent Sc. 2005; 7: 57-60.

10. Portilla-Kirby MJ, Pariona-Minaya MC. Prótesis parcial fija en preescolares. Rev Odontología. 2017; 19 (1): 135-143.

11. Alshiddi IF, Binsaleh SM, Alhawas Y. Patient's perception on the esthetic outcome of anterior fixed prosthetic treatment. J Contemp Dent Pract. 2015; 16 (11): 845-849.

12. Alcaraz Gutiérrez AE, Briones Hernández FM, Vázquez Rodríguez SB, Ruiz Rivera T. Rehabilitación de dientes temporales anterosuperiores con coronas de frente estético, en XXIX Congreso Dental Mundial (FDILA) Riviera Maya, Posgrado de Odontopediatría. 2016.

13. Ramírez Peña HA, Rangel Padilla EE, Martínez-Menchaca HR, Rivera Silva G, Arredondo Campo G, Barba Borrego N et al. Evaluación estética de seis tipos de coronas para dientes primarios. Rev Odontopediatr Latinoam. 2017; 7 (1).

14. García Alonso J, Gutiérrez Oviedo D. Modificación estética de sonrisa utilizando coronas pediátricas NuSmile. Rev Mex Estomatol. 2018; 5 (1): 42-43.

15. Planells del Pozo P, Fuks AB. Zirconia crowns--an esthetic and resistant restorative alternative for ECC affected primary teeth. J Clin Pediatr Dent. 2014; 38 (3): 193-195.

16. Villalobos P. Uso de coronas de zirconio en el tratamiento de caries de la infancia temprana. Odontología Activa Revista Científica. 2018; 2 (2): 23-30.

17. Mondelo LI, Avilés MD, Guarat CMR, et al. Comportamiento de necesidades protésicas en niños. RIC. 2016; 95 (1): 33-42.

18. Gutiérrez Oviedo D, Garza Ayma L, Isassi Hernández H. Coronas de zirconia NuSmile en prótesis infantil: reporte de caso clínico. Rev Mex Estomatol. 2017; 4 (1): 31-32.

19. Cezária Triches T, Barquero Cordero E, Magalhães Benfatti CA et al. Rehabilitación oral con sobredentadura en paciente pediátrico. Odontol Pediátr. 2009; 17 (3): 186-192.

Correspondencia:

Kevin Juárez Casanova

E-mail: kevin_jz@outlook.com

Conflicto de intereses: No existen.

Financiamiento: Ninguno. 